\title{
Prevention and treatment of acute malnutrition in humanitarian emergencies: a multi-organisation collaboration to increase access to synthesised evidence
}

Claire Allen ${ }^{1 *}$, Jeroen Jansen ${ }^{1}$, Celeste Naude ${ }^{2}$, Solange Durao ${ }^{3}$, Monaz Mehta $^{4}$, Erik von Elm5, Saskia van der Kam ${ }^{6}$, Claudine Prudhon ${ }^{7}$, Amy Mayberry ${ }^{8}$, Marie McGrath ${ }^{9}$, Carmelia Alae-Carew ${ }^{10}$, James A. Berkley ${ }^{11}$, Patrizia Fracassi ${ }^{12}$, Nancy Aburto ${ }^{13}$, Mica Jenkins ${ }^{13}$, Jessica Bourdaire ${ }^{13}$, Shona Lang ${ }^{1}$, Alex Nevitte ${ }^{1}$, Isla Kuhn ${ }^{14}$, Shaun Lee ${ }^{15}$ and Hannah Hafezi ${ }^{1,16}$

\begin{abstract}
Background: Program decision-making to prevent and treat acute malnutrition in an emergency can be hampered by a lack of accessible and relevant overviews of directly available robust research evidence. There is often evidence from related settings such as from low-income countries, but this is dispersed across many databases, may be inaccessible and requires assessment of its relevance to the humanitarian setting. We describe a process whereby a multi-disciplinary, international group of specialists worked together to build relevant and effective collections of available systematic reviews on acute malnutrition, published and disseminated as online collections, to improve access to the evidence and concise, synthesised, relevant up to date evidence for programming. By describing this process, we hope to inspire other professional groups to take part in similar multi-stakeholder, multi-disciplinary projects.
\end{abstract}

Objectives: This project was designed to make the evidence from relevant systematic reviews about malnutrition as accessible as possible to support evidence-based decision-making and to guide future research on the prevention and treatment of acute malnutrition in humanitarian emergencies.

Methods: Between March 2017 and March 2018, a large group (21 volunteers and stakeholders) with different backgrounds collaborated to review and curate collections of systematic reviews of interventions for the prevention and treatment of moderate and severe acute malnutrition relevant to humanitarian emergencies. The methodology loosely followed general guidance for overviews of systematic reviews with a pre-defined question (formulated using the PICOS format) and search strategies applied to multiple databases. Pairs of collaborators first screened the search yields to identify potentially eligible reviews, where after other pairs screened the list of potentially eligible reviews for relevance and thus included in the final collections.

\footnotetext{
* Correspondence: callen@evidenceaid.org

${ }^{1}$ Evidence Aid, Oxford, UK

Full list of author information is available at the end of the article
} 
(Continued from previous page)

Results: Search strategies were run in 12 databases, in the week of 15 September 2017, yielding a total of 4646 records after de-duplication. At this point, Cochrane reviews $(n=463)$ and non-Cochrane reviews $(n=4183)$ were separated and handled by different teams to compile three linked collections, namely the Evidence Aid Collection, consisting of relevant non-Cochrane reviews, and two Cochrane Special Collections, consisting of relevant Cochrane reviews, one for prevention and the other for treatment of acute malnutrition. The collections were published on the Evidence Aid website on 12 March 2018 and Cochrane website in August 2018.

Discussion: Through this collaboration, we have successfully generated three collections of systematic reviews to guide prevention and management of acute malnutrition in humanitarian emergencies: an Evidence Aid collection of non-Cochrane reviews, and two Cochrane Special Collections of Cochrane reviews. These collections provide accessible synthesised evidence that can be used to inform decision-making on strategies and policies in the humanitarian emergency and disaster risk reduction sectors and to guide future research by identifying gaps in robust evidence and areas that are under-researched. These collections did not set out to assess methodological quality, appraise in detail what the reviews found or summarise the evidence, but rather to curate the identified relevant systematic reviews into online resources for others to use. This unique collaboration of different individuals, organisations and stakeholders, and the collation of robust evidence can be repeated for other subjects, and Evidence Aid is eager to support new collections around other topics relevant to humanitarian emergencies.

Keywords: Malnutrition, Prevention, Treatment, MAM, SAM, Wasting, Intervention, Review, Humanitarian, Emergency

\section{Background}

Humanitarian emergencies have no single definition but are generally agreed to represent a situation where an event or series of events are a critical threat to the health, safety, security or wellbeing that exceeds the coping capacity of a community or other large group of people (HumanitarianCoalition n.d.; IASC 1994). They may result from internal or external armed conflict, epidemics, famine or natural disasters and usually occurs throughout a large land area.

Local, national and international programming to provide health, nutrition and welfare interventions is necessary in a humanitarian emergency and there is general agreement that this should be based on available best evidence. Primary evidence, such as from randomised controlled trials, may not always be available because of the limited feasibility of this kind of research in humanitarian emergency settings (Bradt 2009). Furthermore, other forms of research undertaken in these settings have previously been noted to be often of weak design, limiting attribution of outcomes to interventions (Blanchet et al. 2017). However, evidence from intervention studies in relevant resource-limited settings may also be of potential value for decision-makers and practitioners, especially where findings are consistent across several studies. Assessment of such literature for likely relevance to humanitarian crises requires subject-specific and context-specific specialist knowledge and is very time-consuming.

A systematic review is a formal study addressing a clearly formulated question using pre-defined and reproducible methods to identify, select and critically appraise all relevant primary studies and analyse pooled data or results from these studies. Systematic reviews are more rigorous than traditional reviews because they seek to minimise bias and statistical imprecision, thus reducing the risk of wrong conclusions, and are well-recognised sources of high-quality evidence (Higgins and Green 2011) (Lavis et al. 2004). There is a growing body of systematic reviews providing syntheses of answers to different types of questions in different settings.

This project was designed to make the evidence from relevant systematic reviews about malnutrition as accessible as possible to support evidence-based decision-making and to guide future research on the prevention and treatment of acute malnutrition in humanitarian emergencies. Evidence Aid led a unique multi-disciplinary collaboration that brought together different stakeholders with varying expertise, including humanitarians, researchers, systematic reviewers, lay-persons, and information specialists that resulted in the publication of three online collections of systematic reviews on prevention and treatment of malnutrition. The process and outcomes of this unique collective effort are shared in this article. By describing this process, we hope to inform and inspire other professional groups to take part in similar multi-stakeholder, multi-disciplinary projects to increase access to information in support of evidence-based programming in the humanitarian sector.

\section{Setting the scene}

In 2017, the United Nations Children's Fund (UNICEF), the World Food Programme (WFP) and other United Nations (UN) agencies estimated that more than 70 million people globally required food or other assistance because of natural disasters, conflict, population displacement, famine or high endemic levels of acute malnutrition (FAO et al. 2017). After an initial decline in humanitarian emergencies following the end of the twentieth century, the number of 
people requiring food and other assistance has dramatically increased. In many situations, the emergency is socio-economic-food is available but unaffordable (Maxwell 2017).

In 2017, in multiple countries, a very large proportion of the population has been affected by humanitarian crises (FEWSNET 2017). Examples include Yemen, where violent conflict and drought has led to an estimated two thirds of the population (more than 17 million people) (UNOCHA 2018f) facing food insecurity and risk of famine across the whole country (Food Security Cluster 2017). This situation is exacerbated by infectious disease outbreaks, including cholera infecting almost 1 million people, breakdown of health service provision and poor access to healthcare services among those affected. In Syria, 7 years of conflict has resulted in an estimated 13 million people in need of humanitarian assistance in appalling conditions, including up to 3 million people trapped in areas beyond the reach of humanitarian assistance (UNOCHA 2018a). Following a devastating famine in Somalia in 2011, a combination of ongoing conflict, the El Niño climatic phenomenon, population displacement and livelihood disruption have resulted in food insecurity among half of its population (UNOCHA 2018e).

In many regions, people have been displaced internally and across borders. Since mid-2017, more than 723,000 Rohingya refugees have fled violence in Myanmar to Bangladesh where they have urgent needs for medical, nutritional, shelter and water and sanitation assistance (UNHCR 2018). In the Democratic Republic of the Congo, it is estimated that more than 4 million people are internally displaced due to armed conflict and insecurity that has persisted for decades (UNOCHA 2018b). In Nigeria, approximately 2 million people have been displaced by conflict, marginalisation and chronic under-development, many across the border to Cameroon, Chad and Niger (UNOCHA 2018c). In South Sudan, nearly 2 million people, $85 \%$ of whom are women and children, have been displaced by conflict, which has now been ongoing for more than 5 years (UNOCHA 2018d).

The right to receive and the obligations to provide, food, nutrition support and medical care in an emergency are clearly enshrined in different bodies of international law, such as humanitarian, human rights and refugee law (ICRC 2005) (FAO 2005). Providing appropriate interventions to support prevention and treatment for child and maternal malnutrition and health can be life-saving. Specific interventions for the prevention and treatment of acute malnutrition have been under development since the 1980s. Guidance and programming of such interventions were traditionally based on observational studies, individual research and expert opinions, but not on evidence systematically collated and analysed (Kerac et al. 2012). Since the 1980s, research and insights have been increasingly generated and are available. High caseloads of acute malnutrition occurring in the context of climatic extremes and military or civil conflict result in a pressing need for curative and preventive interventions that achieve high coverage are effective and cost-effective. To attain these goals, agencies increasingly need to design programmes and interventions based on strong scientific evidence rather than customary practice.

Malnutrition predominantly affects children in the first years of life who are vulnerable from birth because of frequent infectious disease episodes, and as they transition from exclusive breastfeeding through complementary feeding to independent feeding. However, malnutrition may also begin before birth. Low birth weight and premature delivery, as well as posing high risk in themselves, set children on a poor health and nutritional trajectory and are likely determinants of nutritional status in infants and young children. Families affected by humanitarian crises often also face limited access to food, water and sanitation, loss of housing or shelter, an increased rate of infectious diseases requiring medical care, an existing poor health infrastructure and grief, all of which impact the nutritional status of children (Rytter et al. 2014). In addition, low nutritional status makes children susceptible to infections, and, in exchange, infections exacerbate malnutrition (Egal 2006).

\section{Objectives of the project}

The overall aim of the project is to support decision-making with the best available evidence, screened for relevance to the humanitarian sector. Specifically, this project aimed to increase availability and accessibility of robust, synthesised and consolidated evidence by working collaboratively with a large group of multi-disciplinary stakeholders with an interest in nutrition. This evidence will support evidence-based decision-making in programming with a focus on nutrition, to prevent and treat moderate and severe acute malnutrition (MAM and SAM) of vulnerable groups in humanitarian emergencies. The ambition is to maintain a dynamic collection of systematic reviews that can be updated regularly.

\section{Methods}

Between March 2017 and March 2018, the project created three collections of systematic reviews on prevention and treatment of moderate and severe acute malnutrition (MAM and SAM), screened for relevance to humanitarian emergencies, including outcomes relevant to nutrition health. A wide range of stakeholders was convened by Evidence Aid, comprising agency representatives (Action Against Hunger, Cochrane, Cochrane Nutrition, Cochrane Switzerland, Emergency Nutrition Network, KEMRI/Wellcome Trust Research Programme, London School of Hygiene and Tropical Medicine, Médecins Sans Frontières, Save the Children, Scaling up Nutrition (SUN) Movement Secretariat, University of Oxford and the World Food 
Programme) and volunteers (Carmelia Alae-Carew, Jessica Bourdaire, Hannah Hafezi, Isla Kuhn, Shona Lang, Shaun Lee, Alex Nevitte, Beth Sommerville and Georgina Taylor). This collective of key stakeholders agreed on the topic and methods for the collection and supported screening, summarising and categorising of synthesised evidence. The outcomes were made freely accessible as linked collections published on the Evidence Aid website (Evidence Aid 2018) and in the Cochrane Library Special Collections on prevention of acute malnutrition (Cochrane 2018b) and on the treatment of acute malnutrition (Cochrane 2018a).

\section{Scope of the collections}

The collaboration agreed that the scope of the collection would focus on 'The management and prevention of moderate and severe acute malnutrition (MAM and SAM) in humanitarian emergencies'. This topic was partly derived by consulting key stakeholders and informed by an overview of key interventions specified in guidelines, including those from WHO (WHO 2003), the Sphere Handbook (Sphere 2018), and in the Inter-Agency Standing Committee (IASC) Nutrition toolkit (Global Nutrition Cluster n.d.).

Of the 12 areas in the IASC toolkit, we focussed on (but did not limit to) management of MAM, management of SAM and infant and young child feeding in emergencies (given its importance in prevention). We did not focus solely on children and included reviews of evidence in older populations. Management of MAM and SAM included prevention and treatment.

\section{Process, timeline and milestones}

The collaborators agreed on specific steps to guide the process (Fig. 1).

The methodology loosely followed guidance for overviews of systematic reviews (Higgins and Green 2011) with a pre-defined question and search strategies applied to multiple electronic research databases. Firstly, the question was formulated using the PICOS-Population, Intervention, Comparison, Outcomes and Study design format). The PICOS question was used to develop eligibility criteria and detailed search strategies to be implemented in both Cochrane and non-Cochrane databases. Databases searched were the Cochrane Database of Systematic Reviews, MEDLINE, Embase, CINAHL, Global Health, LILACS, ScieLO, PubMed, TRIP, HealthEvidence.org, HealthSystemsEvidence. org, WHO Nutrition and Epistemonikos (see detailed search strategies in Additional file 1).

After de-duplication, all records yielded by the searches were imported into RAYYAN (https://rayyan.qcri.org/welcome) for manual screening using the pre-defined eligibility criteria. The screening was done independently in duplicate and disagreements resolved by discussion or involving a third person. Pairs of collaborators first screened all yielded records to identify potentially eligible reviews, whereafter, other pairs screened all these potentially eligible reviews for

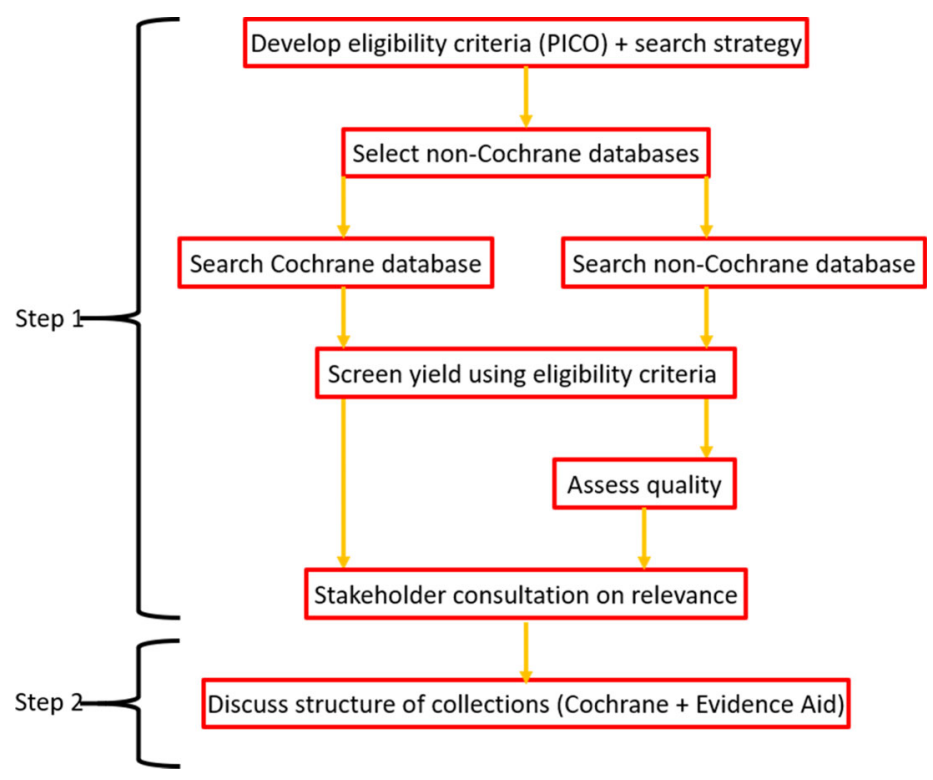

Note: quality of individual reviews was not assessed formally although experts were applying the inclusion/exclusion criteria.

Fig. 1 Steps followed to build the collection of reviews on preventing and treating MAM and SAM*. Note: quality of individual reviews was not assessed formally although experts were applying the inclusion/exclusion criteria 
relevance and thus included in the final collections. We checked the methods of non-Cochrane systematic reviews before inclusion for quality (e.g. at least two online databases had to be included; each review should include a quality assessment of included studies).

Stakeholder consultation took place on the relevance of selected reviews and the final list of reviews for inclusion in collection was generated (including determining the relevance to contexts, such as lower- and middle-income countries, or conflict and disaster-affected countries). The structure of the collections was discussed by the whole group, and one volunteer (BS) prepared brief summaries of all included systematic reviews; these summaries were subsequently translated into French and Spanish. Before publication, one author (SvdK) categorised each review into a topic area based on what was identified. For the Cochrane special collections, included reviews were categorised $(\mathrm{CN})$ drawing on the UNICEF Conceptual Framework of Determinants of Undernutrition (Fig. 2) (UNICEF n.d.), where immediate causes of undernutrition include inadequate dietary intake and disease, and summaries were prepared (MM).

Two authors (JAB and MMG) wrote the introduction for the Evidence Aid collection and Cochrane collaborators wrote introductions for the Cochrane collections. These explained both the purpose and background of the collections. Both collections were published online (Evidence Aid in March 2018 and Cochrane in July 2018) and linked and then disseminated and promoted widely.

\section{Inclusion/exclusion criteria}

The acronym 'PICOS' was used to formulate and develop the research question for the collections: $\mathrm{P}$-patient, problem or population; I-intervention; $\mathrm{C}$-comparison, control or comparator; O-outcome; and S-study design.

The following PICOS was developed and used to define the eligibility (inclusion and exclusion) criteria for the collections:

- Population: populations with or at risk ${ }^{1}$ of moderate and severe acute malnutrition (no age limitations). ${ }^{2}$ We excluded systematic reviews focussed on populations undergoing highly specialised tertiary-level treatments not likely to be available and/or scalable in humanitarian settings; for example, organ and stem cell transplants, most cancer treatments and surgeries, elective rehabilitation and rehabilitative surgeries (e.g. hip replacements) and specialised treatments for chronic liver disease. We also excluded reviews in populations not relevant or specific to humanitarian settings, such as nursing homes, and rehabilitation and specialist treatment centres (e.g. for eating disorders in well-resourced settings).

- Interventions for treatment: treatment (focussed on, but not limited to, infant and young child) included the actual treatment or medical strategy for managing acute malnutrition with direct impact

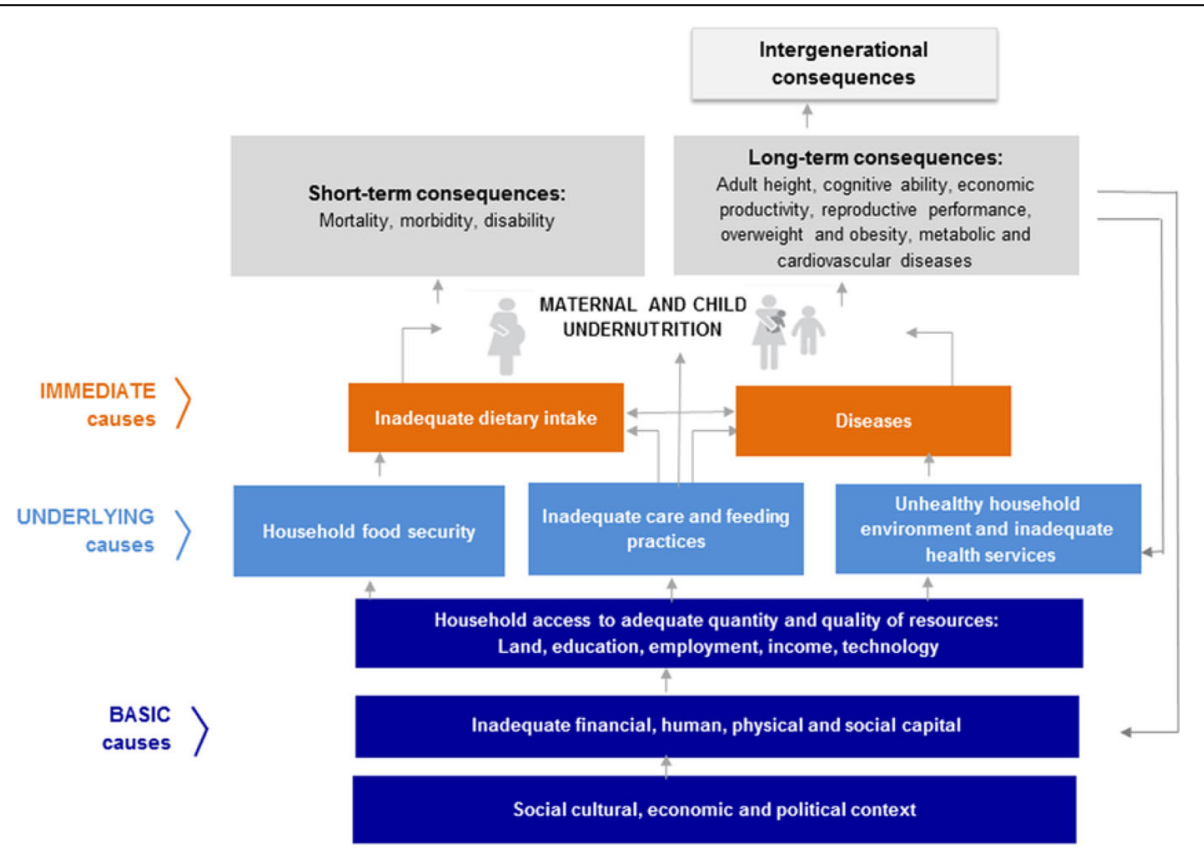

(c) Inclusion/exclusion criteria

Fig. 2 UNICEF conceptual framework for causes of malnutrition (UNICEF n.d.) 
on relevant health outcomes, specifically, mortality, recovery, defaulting, relapse and the occurrence of morbidities.

- Treatment approaches: standard outpatient treatment targeting children 6-59 months of age with low mid-upper arm circumference (MUAC) or low weight-for-height (WHZ) and with good appetite and no medical complications or with oedema + or oedema ++, and inpatient treatment for those with low MUAC (management) or low WHZ (management) and medical complications or poor appetite or with marasmic-kwashiorkor or oedema +++ , children who are $<4 \mathrm{~kg}$, management of children $<6$ months of age with SAM; use of routine antibiotics, vitamin A supplementation in the treatment of children with SAM, and antiretroviral therapy (ART) for the management of HIV-infected children with SAM.

- Intervention duration: activities considered with direct impact on relevant nutrition health outcomes. Depending on the intervention the duration can vary from a few hours (e.g. oral rehydration therapy-ORT) to a few months (e.g. TSFP); in some instances, it is difficult to establish an overall duration. The timescale for measuring outcomes also varies; there is a specific lapse of time to measure each outcome, e.g. TSFP, CMAM, IMAM, micronutrient supplementation, artificial feeding, ORT, IYCF interventions (counselling, messaging, promotion) and other vulnerable group nutrition counselling; infection treatment and management.

- Products and specification for intervention: examples of specific products: ready-to-use therapeutic food (RUTF), F100 and F75 milks, food fortification, micronutrient powders (MNP), ready-to-use supplementary food (RUSF), ready-to-use foods (RUF), lipid-based nutrient supplements (LNS) - according to established specifications, LNS are classified as large quantity (LQ-LNS), medium quantity (MQ_LNS) or small quantity (SQ-LNS) for use in specific target groups/interventions. LQ-LNS are formulated for the management of MAM; MQ-LNS provide macro and micronutrient supplementation to children (6-23 months, 6-36 months) during food insecurity; SQ-LNS primarily provides essential (micro) nutrients intended to children aged 6-23 months).

- Interventions for prevention: activities addressing immediate causes of malnutrition, namely inadequate dietary intake and disease. Examples include micronutrient supplementation, point-of-use fortification, IYCF interventions (counselling, messaging, promotion), and other vulnerable group nutrition counselling; water, sanitation and hygiene (WASH) interventions, vaccinations, specialised food transfers, general food distribution, food fortification, livelihood interventions, cash transfer programmes (CTP), conditional cash transfers, unconditional cash transfers, vouchers, school feeding, anti-anaemia actions, protection of IYCF by safeguarding against inappropriate marketing of breastmilk substitutes, promotion of fruit and vegetable gardens for healthy diets, food safety measures.

- Comparison: variable but could include usual practice depending on the intervention.

- Outcomes: relevant nutrition health outcomes. Examples: recovery, relapse, mortality, morbidity, defaulter, SAM and MAM incidence, adverse events, time to recovery, mean rate of weight gain, anthropometry (e.g. weight gain (total), body mass index (BMI), weight-for-age (WAZ), WHZ, MUAC, BMI-for-age etc.), dietary intake, feeding practices (exclusive breastfeeding, continued breastfeeding, minimum acceptable diet-standard WHO indicators exist), referral to hospitals, recovery from diarrhoea/duration of diarrhoea and cost of treatment per child. Nutritional oedema was included in the context of SAM treatment.

- Study design: Systematic reviews were eligible. No language restriction was applied, and protocols for systematic reviews were excluded. Only reviews published from 2013 onwards were included in the Cochrane collections.

\section{Treatment-examples of interventions}

- Included: community-based Management of Acute Malnutrition (CMAM), Integrated Management of Acute Malnutrition (IMAM), Targeted Supplementary Feeding Programmes (TSFP), micronutrient supplementation, Infant and Young Child Feeding (IYCF), interventions targeting acute malnutrition, Oral Rehydration Therapy (ORT) and infection treatment and management.

- Included in the Cochrane Collection only: treatment of diarrhoea with ORT/zinc, prevention and treatment of vitamin A deficiency, prevention and treatment of micronutrient deficiencies, nutrition, HIV and AIDS as per our eligibility criteria for Treatment "...... with direct impact on relevant health outcomes, .......... and the occurrence of morbidities". They are categorised under the heading: Treatment of disease and conditions with known impacts on nutritional health. 
- Excluded: blanket or general food distribution, the psychosocial components of nutrition, nutritional care for groups with special needs, the use and role of food assistance, food handling, storage and preparation, and household food security and livelihoods, because these were not directly related to health outcomes or medical aspects.

- Excluded from the Evidence Aid collection only: treatment of diarrhoea with ORT/zinc, prevention and treatment of vitamin A deficiency, prevention and treatment of micronutrient deficiencies, nutrition, HIV and AIDS.

Prevention-examples of interventions: prevention was limited to strategies directed at the immediate causes of malnutrition, as defined in the UNICEF conceptual framework (Fig. 2) (UNICEF n.d.).

- Included: micronutrient supplementation, food fortification of staple foods including iodized salt, IYCF and other vulnerable group nutrition counselling, WASH interventions and vaccinations.

- Excluded: food storage/silos support and school feeding.

\section{Supporting documents}

The group identified several relevant documents that individuals used to inform this initiative, as follows:

- A toolkit for addressing nutrition in emergency situations, IASC Global Nutrition Cluster, UNICEF (Global Nutrition Cluster n.d.).

- UNICEF's approach to scaling up nutrition for mothers and their children. Discussion paper (UNICEF 2015).

- Nutrition Cluster Handbook; a practical guide for country-level action, Global Nutrition Cluster (UNICEF 2015).

- Sphere Handbook (Sphere 2018)

- Technical note on supplementary foods for management of MAM; WHO (2012), (WHO 2012).

- Management of severe acute malnutrition in infants and children; WHO (2013), (WHO n.d.).

- Updates on the management of severe acute malnutrition in infants and children. WHO (2013), (WHO 2013).

- The management of nutrition in major emergencies. WHO, UNHCR, IFRC, WFP, (WHO et al. 2000).

\section{Results}

\section{Search results}

Searches (see Additional file 1 for detailed search strategies per database searched) were carried out in 12 databases (see search yields per database in Appendix 1), in the week of 15 September 2017. These searches yielded a total of 4646 potentially eligible papers after de-duplication. At this point, Cochrane reviews $(n=463)$ and non-Cochrane reviews $(n=$ 4183) were separated and handled by different teams to compile the linked collections: Evidence Aid collection, consisting of relevant non-Cochrane reviews, and the Cochrane Special Collections, consisting of relevant Cochrane reviews.

We did not search initially for Kwashiorkor (oedematous malnutrition), but a follow-up literature search and sub-analysis for Kwashiorkor showed that one paper had been omitted from the original search results, which was then included for screening.

Three individuals (CA, JJ and IK) then screened all non-Cochrane titles $(n=4183)$ (see Appendix 2$)$ based on the eligibility criteria, removing any papers that were obviously not related to the collection. Through this process, we identified 1475 potentially eligible records. The 463 Cochrane titles were screened in duplicate by (SVDK, CN, $\mathrm{SD}, \mathrm{MM}, \mathrm{IK})$. The full-text articles of potentially relevant reviews were then screened in duplicate by pairs of reviewers independently (Fig. 3).

\section{Building the collections}

In the Evidence Aid collection, 37 reviews were included initially (with 13 more being included in September 2018). These were grouped under different categories of intervention (topic areas) to help users find reviews of interest (Table 1). In the Cochrane Special Collections, 51 reviews were included, again grouped under different categories of intervention to help users find the reviews of interest (Table 2).

The Evidence Aid collection was first published on 12 March 2018 (Evidence Aid 2018) and currently (18 March 2019) has 49 systematic reviews, mostly freely available. Summaries of reviews have been translated into French and Spanish and uploaded to the collection. More will be uploaded as they are summarised and translated; the aim is to maintain a dynamic collection of systematic reviews which can be updated regularly. For example, on 1 August 2018, 55 papers are eligible to be included (see Appendix 3) and 22 papers remain to be screened.

\section{Discussion and conclusion}

Through this collaboration, we have successfully generated three collections of systematic reviews on prevention and management of acute malnutrition relevant to humanitarian emergencies; an Evidence Aid collection of nonCochrane reviews, and two Cochrane Special Collections of Cochrane reviews. We hope these will inform decisionmaking on strategies and policies in the humanitarian and disaster risk reduction sectors and guide future research by identifying gaps in synthesised evidence and areas that are under-researched. These collections did not set out to 


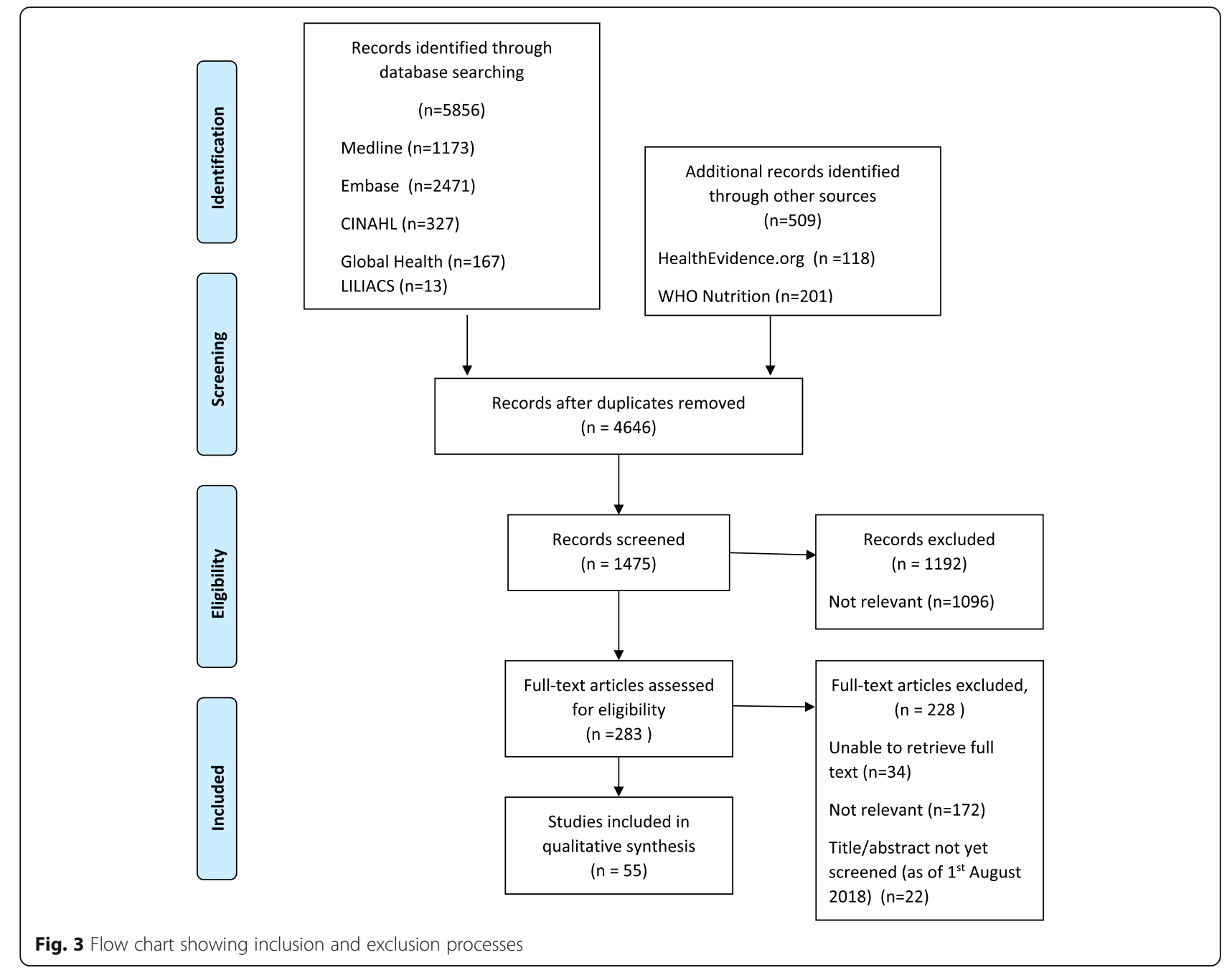

assess methodological quality, appraise in detail what the reviews found or summarise their evidence but rather to curate the identified systematic reviews into accessible online resources for others to use.

Due to the nature of the robust processes within the Cochrane methodology, initially three separate collections have been made available; two of which contain only Cochrane reviews (the Cochrane Special Collections) and one for non-Cochrane reviews (the Evidence Aid evidence collection). It has subsequently been decided to combine the two collections and include the Cochrane reviews in the
Evidence Aid collection. This is to further the aim of this project to collate as much available synthesised evidence on a single platform.

It has been shown that the ready availability of evidence from systematic reviews can be used to inform strategies. As a recent example, a disagreement existed between nutritionists and reproductive health professionals about changing from standard iron supplementation to multiple micronutrient supplementation for pregnant women to prevent anaemia. This disagreement was resolved with the help of the latest systematic review (Haider and Bhutta 2017) published

Table 1 Categories of systematic reviews in the Evidence Aid collection

\begin{tabular}{lc}
\hline Categories of systematic review & Number of reviews included \\
\hline Treatment of malnutrition & 9 \\
Antenatal measures to improve birth outcomes & 10 \\
Breastfeeding promotion & 4 \\
Complementary feeding_improving nutritional status & 11 \\
Disease_-to promote nutrition status in illness & 3 \\
\hline
\end{tabular}


Table 2 Categories of systematic reviews in the Cochrane collection

\begin{tabular}{ll}
\hline Category of systematic review & Number of reviews included \\
\hline Special collection: treatment of acute malnutrition & 2 \\
Treatment of inadequate diet & 5 \\
Treatment of disease and conditions with known impacts on nutritional health & 12 \\
Treatment in preterm, low birth weight infants, and other conditions of foetal growth impairment & 8 \\
Special collection: prevention of acute malnutrition & 12 \\
Prevention of inadequate diet: antenatal interventions & 1 \\
Prevention of inadequate diet: breastfeeding and breastmilk substitutes & 1 \\
Prevention of inadequate diet: in children & 3 \\
Prevention of inadequate diet: in adults & 6 \\
Preventing disease and conditions impacting nutritional health: antenatal and postnatal & 1 \\
Preventing disease and conditions impacting nutritional health: children & 1 \\
Preventing disease and conditions impacting nutritional health: preterm and low birth weight infants; other \\
conditions of foetal growth impairment
\end{tabular}

in the collection. Both supplements showed similar results preventing anaemia, but multiple micronutrient supplementation had additional advantages for the baby. Based on that result, it was decided to also include micronutrients in the supplementation guidance for pregnant women. Having this information in a single source saved time and energy trying to find relevant synthesised evidence.

Systematic reviews are widely regarded as the highest level of scientific evidence, combining results from all available trials on a topic and rigorously assessing their quality. A key limitation is that not all topics have undergone systematic review. Where well-designed trials have not been undertaken, a systematic review may be published as 'empty' or be missing altogether (Yaffe et al. 2012). This may indicate areas where the priorities of decision-makers and practitioners, and those of researchers are not aligned. One important example is the specific treatment of kwashiorkor (Briend 2014).

Some interventions are not amenable to randomisation, and a genuinely informative before/after comparison is challenging to achieve; this is particularly relevant in emergency contexts. Methods for trials and evaluations which compare nutrition alongside other services (e.g. health, water, sanitation and hygiene, and food security) against other nutrition interventions, structured monitoring and evaluation of coverage and performance, for example, as well as qualitative research may provide evidence that could be reviewed; however, the indicators for how to synthesise and systematically measure potential biases and the quality of such qualitative research have only recently been developed (e.g. Grade-Cerqual approach (Evidence Aid 2018)). Qualitative data is often not included in systematic reviews due to the systematic review methodology. This highlights a gap in the research that we have collated within these collections of systematic reviews.
By collating reviews in these focused collections, we hope to also support improved identification of research gaps that are not currently addressed in the existing research.

The collaborative efforts of stakeholders in this project had both advantages and disadvantages. We knew the collection was relevant because the volunteers included those from the humanitarian sector who support both the decision-making process and programme and guideline support. The process took more time than if it had been a funded project, but as a funded project it would be unlikely to have as many active key stakeholders. The disadvantage of working with a volunteer human resource is that not everyone was committed equally, due to competing commitments and limited capacity to contribute to the collective effort; we had to work around existing schedules. However, the positive was that the group worked collaboratively, discussed disagreements and came to a consensus, and there was always an on hand. This collaborative process yielded a strong resource for others that incorporates the wide expertise of this stakeholder group.

It is intended to update the Evidence Aid collection by running the searches on a yearly basis. This will require others to get involved, should the original collaborators not be able to commit to the time required to screen results, etc. There is a limit to what can be sustained on a voluntary basis.

Other areas related to humanitarian emergencies could benefit from consolidated synthesised evidence curated in a single collection to promote evidence-based decision-making. A similar approach to that taken in this collection could be followed for other topics relevant to humanitarian emergencies. Evidence Aid has the expertise in bringing together a collaboration of diverse groups of experts and volunteers, the methodology of developing collections and publishing them on their website. Please contact Evidence Aid (info@evidenceaid.org) if you or your colleagues have an interest in a new collection or would like to take part in updating a collection. 


\section{Appendix 1}

Table 3 Databases searched

\begin{tabular}{ll}
\hline Database & Results \\
\hline Medline via OIVD & 1173 \\
Embase via OVID & 2417 \\
CINAHL via Ebsco & 327 \\
Global Health via Ebsco & 167 \\
LILACS & 13 \\
ScieLO & 31 \\
PubMed & 1110 \\
TRIP & 564 \\
HealthEvidence.org & 209 \\
HealthSystemsEvidence.org & 118 \\
WHO Nutrition & 201 \\
Epistemonikos & 190 \\
Total & 6673 \\
Deduplicated & 4646 \\
\hline
\end{tabular}

\section{Appendix 2}

Table $\mathbf{4}$ Initial screening by title-results

\begin{tabular}{llll}
\hline Total remaining & Allocated & Include & Exclude \\
\hline JJ & 800 & 495 & 305 \\
CA & 1683 & 351 & 1332 \\
IK & 1700 & 629 & 1071 \\
Total & 4183 & 1475 & 2708 \\
\hline
\end{tabular}

\section{Appendix 3}

\section{Full-text screening-results on 1 August 2018}

Full-text screening.

Two hundred thirty-eight full-text papers were selected for screening.

Screening form was completed for each paper by two reviewers:

\author{
Total distributed for double review \\ Running total of included papers to summarising \\ (1 August 2018) \\ Papers for Cochrane Collection (1 August 2018) \\ Number of papers still to be allocated (1 August 2018) \\ [of which guidelines] \\ Number of PDFs not retrieved yet
}

283

55

120

\section{Endnotes}

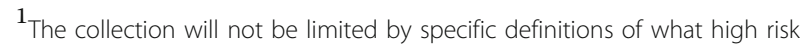
groups are (WHO Guideline: (<-2 WHZ, < $125 \mathrm{~mm} \mathrm{MUAC,} \mathrm{including} \mathrm{both}$ oedema and no oedema, HIV/TB positive, diarrhoea for more than 2 weeks,

etc.), but it will be kept into consideration at a later stage when the collection is collated.

${ }^{2}$ Although it was decided to not include age limitations, the WHO guideline age ranges ( $<6$ months and $6-59$ months) will be kept into consideration at a later stage when the collection is collated.

\section{Additional file}

Additional file 1: Search strategies. (DOCX $38 \mathrm{~kb}$ )

\section{Abbreviations}

ART: Antiretroviral therapy; BMI: Body mass index; CMAM: Community-based management of acute malnutrition; CTP: Cash transfer programmes; HIV: Human immunodeficiency virus; IASC: Inter-Agency Standing Committee; IMAM: Integrated management of acute malnutrition; IYCF: Infant and young child feeding; LNS: Lipid-based Nutrient Supplements; LQ: large quantity; MAM: Moderate acute malnutrition; MMP: Micronutrient powders; MQ: Medium quantity; MUAC: Mid-upper arm circumference; ORT: Oral rehydration therapy; PICO: Patient, intervention, comparison, outcome; PICOS: Patient, intervention, comparison, outcome, study design; RUTF: Ready-to-use therapeutic foods; SAM: Severe acute malnutrition; SQ: Small quantity; TSFP: Targeting supplementary feeding programme; UN: United Nations; UNICEF: United Nations Children's Fund; WASH: Water, sanitation and hygiene; WAZ: Weightfor-age; WFP: World Food Programme; WHO: World Health Organisation; WHZ: Weight-for-height

\section{Acknowledgements}

We acknowledge and thank Beth Sommerville for summarising the included reviews.

\section{Funding \\ No authors received specific funding for this work. \\ Availability of data and materials \\ Not applicable.}

\section{Authors' contributions}

$J$ initiated the project and coordinated it until mid-January 2018, following which CA coordinated the project on behalf of Evidence Aid. CA, JJ, CN, SD, MMehta, SK, CP, AM, MMcGrath, JBerkley, PF, SL and IK, designed the protocol, and when this was complete. IK carried out the literature search and allocated papers to each review author. After screening out papers of no relevance, CA, JJ, CN, SD, MMehta, SK, CP, AM, MMcGrath, JBerkley, JBourdair, PF and SL reviewed abstracts and full texts. JBourdair tagged all reviews according to the Evidence Aid taxonomy. JBerkley and MMcGrath wrote the introduction to the Evidence Aid web-based collection. CA and MMehta made the collections available on the Evidence Aid and Cochrane websites (including translations of the summaries). CA initiated the manuscript based on the design protocol and introduction to the collection and JJ, CN, SD, MMehta, SK, CP, AM, MMcGrath, JBerkley, JBourdaire, PF, $\mathrm{SL}$ and IK contributed to the development of the manuscript. All authors read and approved the final manuscript.

\section{Authors' information}

Not applicable

\section{Competing interests}

The authors declare that they have no competing interests.

\section{Publisher's Note}

Springer Nature remains neutral with regard to jurisdictional claims in published maps and institutional affiliations.

\section{Author details}

${ }^{1}$ Evidence Aid, Oxford, UK. ${ }^{2}$ Centre for Evidence-based Health Care, Stellenbosch University, Stellenbosch, South Africa. ${ }^{3}$ Cochrane South Africa, South African Medical Research Council, Pietermaritzburg, South Africa.

${ }^{4}$ Cochrane, London, UK. ${ }^{5}$ Cochrane Switzerland, Lausanne, Switzerland. ${ }^{6}$ Médecins Sans Frontières, Paris, France. ${ }^{7}$ Save the Children, London, UK.

${ }^{8}$ Action Against Hunger, Paris, France. ${ }^{9}$ Emergency Nutrition Network, Oxford, 
UK. ${ }^{10}$ London School of Hygiene and Tropical Medicine, London, UK. ${ }^{11}$ Univeristy of Oxford, Oxford, UK. ${ }^{12}$ Scaling Up Nutrition (SUN) Movement Secretariat, Geneve, Switzerland. ${ }^{13}$ World Food Programme, Rome, Italy. ${ }^{14}$ University of Cambridge, Cambridge, England. ${ }^{15}$ Monash University (Malaysia), Selangor, Malaysia. ${ }^{16}$ King's College London GKT School of Medical Education, London, UK

Received: 21 December 2018 Accepted: 26 April 2019

Published online: 31 May 2019

\section{References}

Blanchet K et al (2017) Evidence on public health itervention in humanitrian crises. Lancet 390(10109):22787-22296

Bradt DA (2009) Evidence-based decision-making in humanitarian asistance. HPN Network Paper 67. https://www.alnap.org/system/files/content/resource/files/ main/networkpaper067.pdf

Briend, A. (2014) Kwashiorkor : still an enigma-the search must go on. Available at: https://www.ennonline.net/kwashiorkorstillanenigma

Cochrane (2018a) Cochrane special collection: treatment of malnutrition. Available at: https:/www.cochranelibrary.com/collections/doi/10.1002/ 14651858.SC000032/full

Cochrane (2018b) Cochrane special collections: prevention of malnutrition Available at: https://www.cochranelibrary.com/collections/doi/10.1002/ 14651858.SC000031/full

Egal F (2006) Nutrition in conflict situations. Br J Nutr:S17-S19. https://doi.org/10. 1079/BJN20061692

Evidence Aid (2018) Evidence aid nutrition collection. Available at: http://www. evidenceaid.org/prevention-and-treatment-of-acute-malnutrition-inemergencies-and-humanitarian-crises/

FAO (2005) Voluntary guidelines to support the progressive realization of the right to adequate food in the context of national food security, Rome Available at: http://www.fao.org/3/a-y7937e.pdf

FAO et al (2017) The state of food security and nutrition in the world 2017. Building resilience for peace and food security, Rome Available at: https:// www.unicef.org/publications/files/State_of_Food_Security_and_Nutrition_in_ the_World_2017.pdf

FEWSNET (2017) Emergency food assistance needs unprecedented as famine threatens four countries. Available at: http://fews.net/global/alert/january-25-2017

Food Security Cluster (2017) gFSC and gNC event - promoting an integrated famine prevention package: breaking bottlenecks. Available at: http:// fscluster.org/event/gfsc-and-gnc-event-promoting-integrated

Global Nutrition Cluster (n.d.) Toolkit. Available at: http://nutritioncluster.net/ topics/im-toolkit/

Haider B, Bhutta Z (2017) Multiple-micronutrient supplementation for women during pregnancy (review). Cochrane Database Syst Rev 4(4):CD004905. https://doi.org/10.1002/14651858.CD004905.pub5.www.cochranelibrary.com

Higgins, J. and Green, S. (2011) Cochrane handbook for systematic reviews of interventions. Available at: http://handbook-5-1.cochrane.org/

HumanitarianCoalition (n.d.) What is a humanitarian emergency. Available at: https:/www.humanitariancoalition.ca/info-portal/factsheets/what-is-ahumanitarian-crisis

IASC (1994) Definition of complex emergencies; inter-agency standing committee working group XVIth meeting. Available at: https:// interagencystandingcommittee.org/system/files/legacy files/WG16 4.pdf

ICRC (2005) 'Humanitarian law, human rights and refugee law - three pillars'. Available at: https://www.icrc.org/en/doc/resources/documents/statement/ 6t7g86.htm

Kerac, M. et al. (2012) Inpatient treatment of severe acute malnutrition in infants aged <6 months. Available at: http://www.who.int/nutrition/publications/ guidelines/updates_management_SAM_infantandchildren_review9.pdf

Lavis J et al (2004) Use of research to inform public policymaking. Lancet 364: 1615-1621. https://doi.org/10.1016/S0140-6736(04)17317-0

Maxwell D (2017) 21st century famines have nothing to do with a lack of food. In: World economic forum Available at: https://www.weforum.org/agenda/ 2017/03/21st-century-famines-have-nothing-to-do-with-a-lack-of-food

Rytter M et al (2014) The immune system in children with malnutrition-a systematic review. PLoS One 9(8):e105017. https://doi.org/10.1371/journal. pone.0105017 Edited by T. Akiyama

Sphere (2018) The sphere handbook. Available at: https://handbook. spherestandards.org/?string=
UNHCR (2018) Rohingya emergency. Available at: https:/www.unhcr.org/ rohingya-emergency.html

UNICEF (2015) UNICEF's approach to scaling up nutrition for mothers and their children. Available at: https://www.unicef.org/nutrition/files/Unicef_Nutrition Strategy.pdf

UNICEF (n.d.) Unicef conceptual framework. Available at: https:/www.unicef.org/ nutrition/training/2.5/4.html

UNOCHA (2018a) Arab Republic Syrian. Available at: https:/www.unocha.org/syria UNOCHA (2018b) 'Democratic Republic of the Congo (DRC)'

UNOCHA (2018c) Nigeria. Available at: https://www.unocha.org/nigeria

UNOCHA (2018d) Republic of South Sudan

UNOCHA (2018e) Somalia. Available at: https://www.unocha.org/somalia

UNOCHA (2018f) Yemen. Available at: https://www.unocha.org/yemen

WHO et al. (2000) The management of nutrition in major emergencies. Available at: https://www.who.int/nutrition/publications/emergencies/9241545208/en/

WHO (2003) Guidelines for the inpatient treatment of severely malnourished children. Available at: https://www.who.int/nutrition/publications/ severemalnutrition/9241546093/en/

WHO (2012) Supplementary foods for the management of moderate acute malnutrition in infants and children 6-59 months of age; technical note. Available at: https://www.who.int/nutrition/publications/moderate_ malnutrition/9789241504423/en/

WHO (2013) Updates on the management of severe acute malnutrition in infants and children. Available at: https://www.who.int/nutrition/publications/ guidelines/updates_management_SAM_infantandchildren/en/

WHO (n.d.) Management of severe acute malnutrition in infants and children. Available at: https://www.who.int/elena/titles/full_recommendations/sam_ management/en/

Yaffe J et al (2012) Empty reviews: a description and consideration of Cochrane systematic reviews with no included studies. PLoS One. https://doi.org/10. 1371/journal.pone.0036626

\section{Submit your manuscript to a SpringerOpen ${ }^{\circ}$ journal and benefit from:}

- Convenient online submission

- Rigorous peer review

- Open access: articles freely available online

High visibility within the field

- Retaining the copyright to your article

Submit your next manuscript at $\boldsymbol{\nabla}$ springeropen.com 